\title{
A COMPARATIVE STUDY OF VERBAL FLUENCY IN PATIENTS WITH PARANOID SCHIZOPHRENIA, FIRST GRADE RELATIVES AND HEALTHY CONTROLS
}

\author{
Ivanka I. VELEVA ${ }^{1 凶}$, Maya J. STOIMENOVA ${ }^{1}$, Mirena P. VALKOVA ${ }^{2,3}$ \\ ${ }^{1}$ Department of Psychiatry and Medical Psychology, Medical University - Pleven, Pleven, Bulgaria \\ ${ }^{2}$ Clinic of Neurology, University Hospital „Sofiamed“, Sofia, Bulgaria \\ ${ }^{3}$ Psychological department, Philosophic Faculty, University of Veliko Târnovo "St. Kiril and St. Metodi“", \\ Veliko Târnovo, Bulgaria
}

Received 28 Jan 2019, Accepted 03 Apr 2019

hitps://doi.org/10.31688/ABMU.2019.54.2.16

\begin{abstract}
Introduction. Cognitive impairment is a key symptom of schizophrenia and is known to be due to neurodevelopment changes.

The aim of our study was to compare verbal fluency of patients with paranoid schizophrenia (PSch), first grade relatives (FGR) and healthy control subjects $(\mathrm{HC})$ and to find additional risk factors for poor verbal fluency test performances in cases with PSch.

Material and methods. 108 PSch (66 males, $44 \mathrm{fe}-$ males, mean age $38.86 \pm 10.02$ y), 58 FGR ( 30 males, 28 females, mean age $36.71 \pm 11.74$ y) and $60 \mathrm{HC}(37$ males, 23 females, mean age $35.68 \pm 11.36$ y) were studied during the period 2015-2017 at the Department of Psychiatry and Medical Psychology, University Hospital Pleven, Bulgaria. After giving informed consent, they underwent full somatic and neurological examinations and fulfilled Isaac's Set Test (IST) and K, A, M, E literal fluency test (LF).

Results. PSch obtained lower scores on both LF and IST than other two groups. FGR had poor performance only on LF. Ageing, level of formal education,

\section{RÉsumé}

Etude comparée des résultats des tests de l'expression verbale facile chez les patients avec de la schizophrénie paranoïde, de parents au premier degré et de témoins sains

Introduction. Les troubles cognitifs de la schizophrénie sont un domaine d'intérêt scientifique depuis les années 70 du XX siècle. Ils constituent un symptôme clé et sont discutés en relation avec l'hypothèse d'un développement neurologique altéré dans la schizophrénie. Le but de notre étude est de comparer les résultats des tests de la facilité verbale des patients atteints de schizophrénie paranoïde (PCh), de primo-nés $(\mathrm{PN})$ et de témoins sains (TS).

Matériel et méthodes. L'étude a été menée sur 108 patients atteints de (PCh) - (66 hommes et 42 femmes, d'âge moyen à $38,86 \pm 10,02), 58$ patients (PN) $(30$ hommes et 28 femmes, à un âge moyen de 36,71 \pm 11,74) et 60 IC. 23 femmes, d'âge moyen 35,68 211,36 ) au moyen du test de Isaac (IST) pour la sémantique et le type FL K-A-M-E.
\end{abstract}


severity and duration of schizophrenia were associated with LF and IST performances.

Conclusions. PSch show significant LF and semantic fluency difficulties. FGR have only LF changes. Verbal fluency is related to ageing, formal education, duration and severity of schizophrenia.

Keywords: verbal fluency, literal fluency, semantic fluency, schizophrenia, first grade relatives.

\section{INTRODUCTION}

Schizophrenia is a severe, chronic psychiatric disorder, characterized by changes in almost all psychic spheres, loss of personal unity and abnormal interpretation of reality. It is associated with significant social and professional dysfunction.

Rich evidence on specific cognitive dysfunction, not only as epiphenomenon due to therapy but core feature of the disease is currently been collected ${ }^{1}$. These cognitive changes have been discussed in association with neurodevelopmental theory and changes in brain circles, engaged in cognition, particularly these, related to prefrontal cortex and its networks to cortical and subcortical structures ${ }^{2,3}$.

Cognitive changes in schizophrenia are known to be developed at early stages of the disease and even before the first psychotic episode ${ }^{4,5}$. They remain comparatively stable over time and probably are observed in relatives at lower degrees ${ }^{4}$.

Basic executive dysfunction (particularly in attention, psychomotor speed, inhibitory control) seems to be crucial for the whole cognitive impairment due to schizophrenia ${ }^{6}$. It can be manifested in verbal fluency (VF) difficulties.

The AIM OF OUR STUDY was to compare VF of patients with paranoid schizophrenia (PSch), first grade relatives (FGR) and healthy control subjects (HC) and to find additional risk factors for poor VF test performances in cases with PSch.

\section{Material AND Methods}

A total of 226 subjects, divided into three groups, were studied in our research, during the period 2015-2017, at The Department of Psychiatry
Résultats. Les patients atteints de PCI présentaient des résultats inférieurs aux deux tests à la fois en IST et en $\mathrm{FL}$, par rapport à $\mathrm{PR}$ et au $\mathrm{PC}$, ainsi que des violations de la qualité des mots générés. Les IP montrent une FL inférieure à celle du CI. L'influence sur le succès des TSI et de la FL chez PSI est liée à l'âge, à l'éducation, aux années de maladie et à la gravité de la maladie, ainsi qu'à la gravité des symptômes négatifs et des symptômes de désorganisation.

Conclusions. Les PCh ont montré des anomalies significatives de la FL et une facilité sémantique quantitative et qualitative. Les PN montrent une FL cassée.

Mots-clés: expression verbale facile, facilité littérale, facilité sémantique, schizophrénie, parents au premier degré.

and Medical Psychology, University Hospital Pleven, Bulgaria. Of them, 108 (66 males and 42 females, mean age $38.86 \pm 10.02$ y) had PSch, according to the criteria of International Classification of Diseases-10 and met the following additional criteria: (1) disease duration of minimum 1 year; (2) on regular and dose-adequate antipsychotic treatment; (3) on stable state without exacerbations during the last 3 months before examination; (4) with clinically significant schizophrenia symptoms defined as minimum "mild", according to the Positive and Negative Syndrome Scale (PANSS) and with disease severity measured at least "mild" on Clinical global impression (CGI) and (5) without any other significant somatic or brain diseases, substance dependence or mental retardation.

We recruited 58 otherwise healthy FGR (30 males, 28 females, mean age $36.71 \pm 11.74 y)$ and 60 HC (37 males, 23 females, mean age 35.68 $11.36 y$ ).

All examined subjects were fluent in Bulgarian language, with minimal formal education of 8 years and without any sensory deficit which could impact on VF and Isaac's Set Test (IST) performances.

After giving informed consent, all subjects who fulfilled the study criteria underwent full somatic and neurological examinations and clinical interview (incl. family and medical history). The clinical state of PSch was evaluated using PANSS and CGI. Positive, negative syndromes and disorganization were assessed by PANSS subscales. All of recruited subjects were examined via IST-test for semantic verbal fluency and 4 minutes K-A-M-E test for literal fluency (LF) in Bulgarian language.

Intergroup results were compared via multiple sample comparison tests (on the basis of ANOVA and Fisher's tests), using EPSS 20 and Statgraphics plus 5.0. Qualitative analysis of differences between generated words was additionally done on the basis of word 
Table 1. Demographics of the group of study.

\begin{tabular}{|c|c|c|c|}
\hline Groups & PSch & FGR & $\mathrm{HC}$ \\
\hline $\mathrm{N}=$ & 108 & 58 & 60 \\
\hline Age (y) & $38.86 \pm 10.02$ & $36.71 \pm 11.74$ & $35.68 \pm 11.36$ \\
\hline Sex (males:females) & $66: 42$ & $30: 28$ & $37: 23$ \\
\hline $\begin{array}{c}\text { Formal education } \\
\text { (basic 8y; middle 9-12y; high }>12 y \text { ) }\end{array}$ & $24 ; 53 ; 31$ & $4 ; 33 ; 21$ & $7 ; 35 ; 18$ \\
\hline
\end{tabular}

Legend: PSch paranoid schizophrenia, FGR - first grade relatives, HC - healthy controls; y-years

Table 2. Differences between patients with paranoid schizophrenia, first grade relatives and healthy controls.

\begin{tabular}{cccccc}
\hline Type of verbal fluency & PSch & FGR & HC & $\mathrm{p}_{\text {(PSch-FGR) }}$ & $\mathrm{p}_{\text {(FGR-HC) }}$ \\
\hline $\begin{array}{c}\text { IST - semantic fluency } \\
\text { (average points) }\end{array}$ & 28.59 & 32.91 & 34.38 & 0.0001 & $>0.05$ \\
\hline $\begin{array}{c}\text { LF - literal fluency } \\
\text { (average points) }\end{array}$ & 18.05 & 21.66 & 23.25 & 0.0001 & 0.0021 \\
\hline
\end{tabular}

Legend: PSch paranoid schizophrenia, FGR - first grade relatives, HC - healthy controls; IST - Issac's Set Test, LF - literal fluency test

cluster frequency and ability to generate words from different word clusters.

We used regression analysis (linear models) for finding relations between specific factors (education, sex, ageing, PANSS, duration of schizophrenia) and IST and LF performances. All results were interpreted at $95.0 \%$ confidence level.

\section{Results}

Demographics. Demographic data from three examined groups are summarized in Table 1. Mean duration of PSch (from the first episode to the study) was $12.8 \pm 8.2$ years. The average disease severity, measured by PANSS, was $71.8 \pm 5.1$ points.

IST and LF performances. Summary of VF test results (IST and LF) is given in Table 2. PSch group results on both scales were significantly lower than those obtained from the other two; however, FGR and HC showed difference only in LF, but not IST. $44.9 \%$ of PSch performed well on IST and $29.1 \%$ on LF.

Unlike FGR and HC, 36\% of PSch generated words of low cluster frequency (e.g. "mammoth", "dragon", "tyrannous", maurakuya, litchi, Pompey, Machu Picchu, etc.), $75 \%$ of them had difficulties in cluster switching and one third listed only predators or reptiles on IST animal subcategory.

The influence of additional factors on test performances of patients with PSch. Positive correlations were found between education and IST/LF performances $(p=0.0001)$. Ageing had negative impact on IST ( $r r=-0.22 ; p=0.0225)$, but not on LF ( $>>0.05)$. There were no statistically significant sex differences.
IST performance was related to disease duration $(\mathrm{rr}=-0.22 ; \mathrm{p}=0.0120)$ and severity, measured by PANSS ( $r r=-0.49 ; \mathrm{p}=0.0001$ ).

PSch with severe positive symptoms used less word clusters and had difficulties in cluster switching. The degrees of negative symptoms and symptoms of disorganization worsened IST performance $(\mathrm{rr}=-0.47$; $\mathrm{p}=0.0001 ; \mathrm{rr}=-0.46 ; \mathrm{p}=0.0001$, respectively).

Disease duration and severity were related to LF performance $(r r=-0.22 ; p=0.0224 ; r r=-0.42$; $\mathrm{p}=0.0001$, respectively). The most important roles on LF scores played the degree of negative symptoms $(\mathrm{rr}=-0.50 ; \mathrm{p}=0.0001)$ and symptoms of disorganization $(\mathrm{rr}=-0.53 ; \mathrm{p}=0.0001)$.

\section{Discussion}

Our analysis supports the previous findings of significant semantic and LF impairment related with $\mathrm{PSch}^{7-9}$. There are two main hypotheses that have been used for explanation of the development of VF impairment due to PSch: the first is pointed on semantic storage disorganization ${ }^{8}$; the second on difficulties in word extraction from memory depot as a specific expression of wide intellectual deficit? According to Rinaldi et $\mathrm{al}^{8}$, such deficit has been associated with specific impairment on semantic level. However, we suppose not semantic memory, but executive dysfunction plays the main role for VF deficit. Semantic fluency is known to be mainly related to temporal lobe functions and networks. Unlike semantic, LF performance is related predominantly with frontal lobe ${ }^{10}$ which is strongly associated with neurodevelopment changes in PSch ${ }^{2,3}$. LF compared to semantic requires more complex operations in 
word finding (use of great number of word clusters from different word trees), more mental operations, higher psychomotor speed and faster and accurate interactions between temporal and frontal lobe. Qualitative analysis of words meaning gives additional information for frontal and temporal lobe dysfunctions related to VF. PSch patients tend to generate low-frequency words (having difficulties in word-tree arrangement and hierarchy) from small number of word clusters (impaired information procession speed). One third of them use words with frightening meaning, probably due to defective thinking and reflection of themselves and environment ${ }^{11}$, egocentrism and replacing of positive emotions by negative affect and anger. Such findings suspect significant loss of frontal control over affective domains. Moreover, PSch shows tendency for generating words related to mythology and history, but not to nearby environment and reality. The possible explanation can be searched in "loose of associations " 7,12 phenomenon, which is strongly related to specific impairment of inhibitory control (basic frontal function). Another possible explanation of impaired VF is searched in association with schizophrenia related low cognitive reserve ${ }^{13}$. FGR show normal IST scores but have lower LF performance compared to HC, though significantly better than PSch. Such subjects can compensate the basic cognitive deficit by using a lot of cognitive capacity in semantic fluency tasks, but fail in more complex tasks like LF. However, they manage to suppress irrelevant to reality and environment associations and remain capable to use high-frequency words from different word clusters. So, FGR differentiate from $\mathrm{HC}$ in these specific cognitive constructs in which PSch show most obvious deficits ${ }^{14}$. We suggest that VF (especially LF) can be used as endo-phenotype marker for schizophrenia.

Ageing and education have probably the biggest impact on VF in population based studies ${ }^{15,16}$. We obtained mild but significant decline of LF scores with ageing in patients with PSch. It can be explained by age-associated processing speed delay, which concerned mainly frontal functions. However, low education itself remains stable risk-factor for VF dysfunction even in cases with PSch. Egeland J. et $\mathrm{al}^{17}$ have obtained gender differences in VF of general population (especially for word meaning). Unlike them, we fail to find any sex differences in PSch group.

Disease duration and severity are significantly related to PSch VF performances ${ }^{18-20}$, though different disease signs have various effects. Possible symptoms have shown no significant association with cognitive decline $^{18}$, including LF, although some mild difficulties are obtained in word cluster formation. Unlike them, negative symptoms and disorganization have strong impact on $\mathrm{VF}^{18-20}$. It suggests different models of prefrontal and frontal circuit functioning between PSch with more prominent positive or negative/disorganization signs.

\section{Conclusions}

PSch shows significant LF and semantic fluency difficulties. FGR have only LF changes. VF is related to ageing, formal education, duration and severity of schizophrenia.

\section{Compliance with Ethics Requirements:}

„The authors declare no conflict of interest regarding this article"

„The authors declare that all the procedures and experiments of this study respect the ethical standards in the Helsinki Declaration of 1975, as revised in 2008(5), as well as the national law. Informed consent was obtained from all the patients included in the study"

"No funding for this study"

\section{References}

1. Breier A. Cognitive deficit in schizophrenia and its neurochemical basis. Br J Psychiatry. 1999;(37):16-8.

2. Anjomshoa A, Dolatshahi M, Amirkhani F, Rahmani F, Mirbagheri M, Aarabi M. Structural brain network analysis in schizophrenia using minimum spanning tree. Conf Proc IEEE Eng Med Biol Soc. 2016:4075-4078.

3. Weinberger D. Future of days past: neurodevelopment and schizophrenia. Schizophr Bull. 2017;43(6):1164-1168.

4. Erlenmeyer-Kimling L. Neurobehavioral deficits in offspring of schizophrenic parents: liability indicators and predictors of illness. Am J Med Genet. 2000;97(1):65-71.

5. Cuesta M, Moreno-Izco L, Ribeiro M, et al. Motor abnormalities and cognitive impairment in first-episode psychosis patients, their unaffected siblings and healthy controls. Schizophr Res. 2018;200:50-55.

6. Kar S, Jain M. Current understandings about cognition and the neurobiological correlates in schizophrenia. J Neurosci Rural Pract. 2016;7(3):412-8.

7. Bokat C, Goldberg T. Letter and category fluency in schizophrenic patients: a meta-analysis. Schizophr Res. 2003; 64(1): 73-8.

8. Rinaldi R, Lefebre L, Trappeniers J. Language, executive functioning and symptomatology - Is fluency a trasversal tool in schizophrenia? Open J of Psychiatry. 2013;3:358-69.

9. Shao Z, Janse E, Visser K, Meyer A. What do verbal fluency tasks measure? Predictors of verbal fluency performance in older adults. Front Psychol. 2014; 5: 772.

10. Bozikas V, Kosmidis M, Karavatos A. Disproportionate impairment in semantic verbal fluency in schizophrenia: Differential deficit in clustering. Schizophr Res. 2005;74(1):51-9.

11. Lezak M, Howieson D, Loring D, Hannay H, Fischer J.

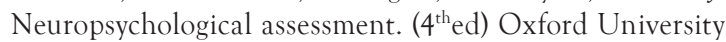
Press, 2004. p.1016. 
12. Troster A, Fields J, Testa J, et al. Cortical and subcortical influences on clustering and switching in the performance of verbal fluency tasks. Neuropsychologia 1998;36(4):295-304.

13. De la Serna E, Andres-Perpina S, Puig O, et al. Cognitive reserve as a predictor of two year neuropsychological performance in early onset first-episode schizophrenia. Schizophrenia Res. 2013; 143(1):125-31.

14. Appels MC, Sitskoorn MM, Westers P, Lems E, Kahn RS. Cognitive dysfunctions in parents of schizophrenic patients parallel the deficits found in patients. Schizophr Res. 2003;63(3):285-93.

15. Ratcliff G, Dodge H, Birzescu M, Ganguli M. Tracking cognitive functioning over time: Ten-year longitudinal data from a community-based study. Appl Neuropsychol. 2003;10:76-88.

16. Van Der Elst W, Van Boxtel M, Van Breukelen G, Jolles J. Normative data for the Animal, Profession and Letter M Naming verbal fluency tests for Dutch speaking participants and the effects of age, education, and gender. $J$ Int Neuropsychol Soc. 2006;12:80-9.
17. Egeland J, Landro N, Tjemsland E, Walbakken K. Norwegian norms and factor-structure of phonemic and semantic word list generation. Clin Neuropsychologist. 2006; 20:716-72.

18. O'Leary D, Flaum M, Kesler M, Flashman L, Arndt S, Andreasen N. Cognitive correlates of the negative, disorganized, and psychotic symptom dimensions of schizophrenia. J Neuropsychiatry Clin Neurosci. 2000;12(1):4-15.

19. Brébion G, Villalta-Gil V, Autonell J, et al. Cognitive correlates of verbal memory and verbal fluency in schizophrenia, and differential effects of various clinical symptoms between male and female patients. Schizophrenia Research. 2013;147:81-5.

20. Wu Z, Yang Z, Zhang M, Bao X, Han F, Li L. The role of $\mathrm{N}$-methyl-D-aspartate receptors and metabotropic glutamate receptor 5 in the pre pulse inhibition paradigms for studying schizophrenia: pharmacology, neurodevelopment, and genetics. Behav Pharmacol. 2018;29(1):13-27. 